УДК 532.5.013.4

\title{
The Influence of Changes in the Internal Energy of the Interface on a Two-Layer Flow in a Cylinder
}

\author{
Evgeniy P. Magdenko* \\ Institute of Computational Modelling SB RAS \\ Academgorodok, 50/44, Krasnoyarsk, 660036 \\ Institute of Mathematics and Computer Science \\ Siberian Federal University \\ Svobodny, 79, Krasnoyarsk, 660041 \\ Russia
}

Received 10.05.2018, received in revised form 10.06.2018, accepted 20.11.2018

The exact solution of the equations of the creeping flow model with the Himentsa type velocity field is considered in this paper. The solution describes thermocapillary convection in layers. It is interpreted as the motion of viscous heat-conducting liquids in a cylinder with solid walls and combined movable non-deformable interface. At the same time there are no mass forces. From a mathematical point of view the resulting initial-boundary problem is inversible and nonlinear because the total energy condition at the interface is taken into account. It is established that there can be two such solutions.

Keywords: nonlinear inverse problem, Marangoni number, energy condition, creeping thermocapillary motion, Himentsa solution.

DOI: $10.17516 / 1997-1397-2019-12-2-213-221$.

The specificity of the phenomena occurring at the phase interface is associated with the existence of energy and entropy of the surface phase which are excessive with respect to the volumetric phases in the phase transition layer [1]. However, the energy exchange between the volumetric and surface phases is not well studied. For ordinary fluids at room temperature, the effect of changes of the internal energy of the interfacial surface on the formation of heat fluxes, temperature fields and velocities in the surface vicinity is insignificant in comparison with viscous friction and heat transfer [2]. Little attention has been given to the class of problems associated with these phenomena.

When a liquid media with an interface moves in an inhomogeneous temperature field the difference in heat fluxes is not equal to zero [3],

$$
k_{2} \frac{\partial \theta_{2}}{\partial n}-k_{1} \frac{\partial \theta_{1}}{\partial n}=æ \theta \operatorname{div}_{\Gamma} \mathbf{u}+\omega\left(\theta_{t}+\mathbf{u} \cdot \nabla_{\Gamma} \theta\right),
$$

where $æ=-\partial \sigma / \partial \theta, \omega=\partial(\sigma(\theta)+æ \theta) / \partial \theta, \sigma(\theta)$ is the surface tension coefficient. In relation (1) $k_{j}$ is the coefficient of heat conduction, $\theta_{j}$ is the temperature of a liquid, $j=1,2 ; \theta=\theta_{1}=\theta_{2}$, $\mathbf{u}=\mathbf{u}_{1}=\mathbf{u}_{2}$ are common temperatures and velocity vectors at the interface $\Gamma$ and $\mathbf{n}$ is the normal to $\Gamma$ directed to the second fluid. For many liquids, $\sigma(\theta)$ is a linear function of temperature

$$
\sigma(\theta)=\sigma_{0}-æ\left(\theta-\theta_{0}\right)
$$

*magdenko_evgeniy@icm.krasn.ru

(c) Siberian Federal University. All rights reserved 
where $\sigma_{0}, æ, \theta_{0}$ are constants. In this case energy relation (1) is simplified:

$$
k_{2} \frac{\partial \theta_{2}}{\partial n}-k_{1} \frac{\partial \theta_{1}}{\partial n}=æ \theta \operatorname{div}_{\Gamma} \mathbf{u}
$$

The ratio of the right-hand side of (3) to the first term in the left-hand side of (3) is $\mathrm{E}=$ $=\mathfrak{x}^{2} \theta^{*} / k_{2} \mu_{2}$. Here $\mu_{j}$ is the dynamic viscosity, and $\theta^{*}$ is the characteristic temperature at the interface. This value characterizes the significance of the process of releasing or absorption of heat during local increments of the interfacial surface for the development of convective motion near the interface. For ordinary liquids the value of $\mathrm{E}$ is small at room temperature, and changes in the characteristic velocity of convection due to increments of surface internal energy are insignificant [2]. For example, experiments for the water-ethyl alcohol system at $\theta^{*}=15^{\circ} C$ give $\mathrm{E} \sim 5 \cdot 10^{-4}$. Therefore, the right-hand side in (1) is often omitted, and we have the equality of heat fluxes across the interface.

However, at sufficiently high temperatures when viscosity and thermal conductivity of ordinary liquids are significantly reduced and for liquids with reduced viscosity (for example, for some cryogenic liquids, such as liquid $\mathrm{CO}_{2}$ ) effects associated with the heat of formation of the interfacial surface can have a significant impact on fluid motion [4]. The maximum value of parameter $\mathrm{E}$ is reached near the critical points. It is known that for water $\mathrm{E} \sim 0.02$ at $\theta=303.15 \mathrm{~K}$; $\mathrm{E} \sim 0.6$ at $\theta=573.15 \mathrm{~K} ; \mathrm{E} \sim 0.7$ at $\theta=623.15 \mathrm{~K}$ (the critical value for water is $\theta_{\mathrm{cr}}=647.3 \mathrm{~K}$ ). Let us note that mechanism of local changes in the internal energy of an interfacial surface (IEIS) affects the convective stability of thermocapillary systems (see [3], chapter VI). This mechanism should be taken into account for liquids at elevated temperatures and low viscosity. In particular, it was shown that the increment of IEIS leads to the expansion of the stability limits at rest of a horizontal flat layer of liquid in the presence of a temperature gradient in the vertical direction. The effect of IEIS changes on the linear and weakly nonlinear stability of the two-layer Poiseuille flow and film flow of a low-viscosity fluid for an isothermal ground state shows up as the change in the phase velocity of waves. In all considered problems the ground state did not depend on the IEIS.

The exact solution for the equations of a creeping-flow model with a velocity field of the Himents type is obtained in this paper. It describes thermocapillary convection in a two-layer viscous heat-conducting fluid. The motion occurs in a cylinder with solid walls and a common movable non-deformable interface. In this case mass forces are absent. From a mathematical point of view the initial boundary-value problem is inversible and nonlinear because the total energy condition at the interface is taken into account. It is established that there can be two such solutions.

\section{The problem formulation and its transformation}

The equations of rotationally symmetric stationary motion of a viscous incompressible heatconducting fluid in the absence of mass forces have the form

$$
\begin{gathered}
u u_{r}+w u_{z}+\frac{1}{\rho} p_{r}=\nu\left(\Delta u-\frac{u}{r^{2}}\right) \\
u w_{r}+w w_{z}+\frac{1}{\rho} p_{z}=\nu \Delta w \\
u_{r}+\frac{1}{r} u+w_{z}=0 \\
-214-
\end{gathered}
$$




$$
u \theta_{r}+w \theta_{z}=\chi \Delta \theta
$$

Here $u(r, z), w(r, z)$ are components of the velocity vector on the axes $r$ and $z$ of the cylindrical coordinate system, $p(r, z)$ is pressure, $\theta(r, z)$ is fluid temperature; $\rho, \nu, \chi$ are the density, the kinematic viscosity and the thermal diffusivity, respectively; $\Delta=\partial^{2} / \partial r^{2}+r^{-1} \partial / \partial r++\partial^{2} / \partial z^{2}$ is the Laplace operator.

The solution of system (4)-(7) is taken in the form

$$
u=u(r), \quad w=v(r) z, \quad p=p(r, z), \quad \theta=\theta(r, z) .
$$

This is an axisymmetric analog of the Himenz solution. The substitution of expressions for $u, w$ и $p$ from (8) in the equations of motion (4), (5) leads to the following relations

$$
\begin{gathered}
\frac{1}{\rho} p_{r}=\nu\left(u_{r r}+\left(\frac{1}{r} u\right)_{r}\right)-u u_{r}, \\
\frac{1}{\rho} p_{z}=\left(u v_{r}+v^{2}-\nu\left(v_{r r}+\frac{1}{r} v_{r}\right)\right) z .
\end{gathered}
$$

It follows from the analysis of the obtained expressions that $p_{r}$ is a function of variable $r$, and $p_{z}$ linearly depends on variable $z$. Taking into account (8), we obtain from equations of motion (4)-(6) that

$$
\begin{gathered}
u_{r}+\frac{1}{r} u+v=0, \quad u v_{r}+v^{2}=\nu\left(v_{r r}+\frac{1}{r} v_{r}\right)+f \\
\frac{1}{\rho} p=d(r)-\frac{f}{2} z^{2}, \quad d=\nu\left(u_{r}+\frac{1}{r} u\right)-\frac{1}{2} u^{2}+d_{0}, \quad d_{0}=\text { const }
\end{gathered}
$$

where $f$ is an arbitrary constant that is the pressure gradient along the axis of the cylinder.

Equation for temperature (7) can be rewritten as

$$
u(r) \theta_{r}+v(r) z \theta_{z}=\chi \Delta \theta .
$$

One of the solutions of this equation is quadratic with respect to the variable $z$ function of the form

$$
\theta(r, z)=a(r) z^{2}+b(r)
$$

Thus the temperature takes an extreme value at point $z=0$. It has maximum value when $a(r)<0$, and it is minimum value when $a(r)>0$.

Let us apply solutions (9), (10) to describe the two-layer motion of viscous heat-conducting fluids in the cylinder $0 \leqslant r \leqslant R_{2}$ with the solid wall at $r=R_{2}=$ const and with the cylindrical interface at $r=R_{1}, 0<R_{1}<R_{2}$. The fluid 1 occupies the region $0 \leqslant r \leqslant R_{1}$, and the fluid 2 occupies the cylindrical layer $R_{1} \leqslant r \leqslant R_{2}$ (Fig. 1). Parameters of fluids are $\rho_{j}, \nu_{j}, \chi_{j}, j=1,2$.

Substituting (9), (10) into equations of motion (4)-(6) and heat transfer equation (7), we can obtain that $u_{j}(r), v_{j}(r), a_{j}(r), b_{j}(r)$ are solutions of the following systems of equations $(j=1,2)$

$$
\begin{gathered}
u_{j} v_{j r}+v_{j}^{2}=\nu_{j}\left(v_{j r r}+\frac{1}{r} v_{j r}\right)+f_{j}, \\
u_{j r}+\frac{1}{r} u_{j}+v_{j}=0 . \\
2 v_{j} a_{j}+u_{j} a_{j r}=\chi_{j}\left(a_{j r r}+\frac{1}{r} a_{j r}\right), \\
u_{j} b_{j r}=\chi_{j}\left(b_{j r r}+\frac{1}{r} b_{j r}\right)+2 \chi_{j} a_{j}, \\
-215-
\end{gathered}
$$




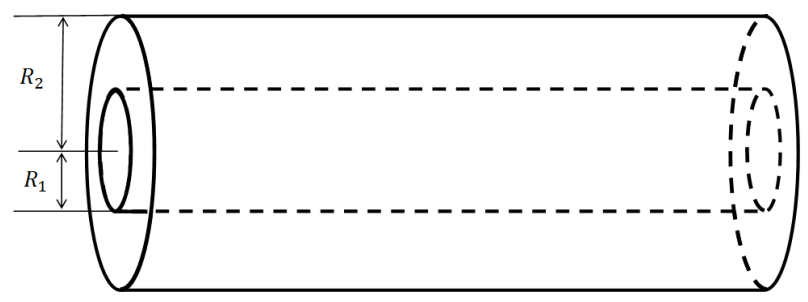

Fig. 1. Cylindrical domain with interface

The pressure obeys the following equation

$$
\frac{1}{\rho_{j}} p_{j}(r, z)=d_{j}(r)-\frac{f_{j}}{2} z^{2}, \quad d_{j}=\nu\left(u_{j r}+\frac{1}{r} u_{j}\right)-\frac{1}{2} u_{j}^{2}+d_{j 0}, \quad d_{j 0}=\text { const. }
$$

On solid wall at $r=R_{2}$ we have conditions

$$
\begin{array}{cc}
u_{2}\left(R_{2}\right)=0, & v_{2}\left(R_{2}\right)=0, \\
a_{2}\left(R_{2}\right)=\alpha, & b_{2}\left(R_{2}\right)=\beta,
\end{array}
$$

with given constants $\alpha, \beta$. Let us note that for $\alpha<0$ the temperature on the wall has maximum value at $z=0$ and for $\alpha \geqslant 0$ it has minimum value at $z=0$.

Taking into account the temperature dependence of the surface tension coefficient $\left(\sigma=\sigma_{0}-\right.$ $\left.-\rightsquigarrow\left(\Theta-\Theta_{0}\right)\right)$ and $(10)$, we obtain at the interface $r=R_{1}$ the following conditions

$$
\begin{gathered}
u_{1}\left(R_{1}\right)=u_{2}\left(R_{1}\right)=0, \quad v_{1}\left(R_{1}\right)=v_{2}\left(R_{1}\right), \\
\mu_{2} v_{2 r}\left(R_{1}\right)-\mu_{1} v_{1 r}\left(R_{1}\right)=-2 æ a_{1}\left(R_{1}\right), \\
\left.a_{1}\left(R_{1}\right), t\right)=a_{2}\left(R_{1}\right), \quad k_{2} a_{2 r}\left(R_{1}\right)-k_{1} a_{1 r}\left(R_{1}\right)=æ a_{1}\left(R_{1}\right) v_{1}\left(R_{1}\right), \\
\left.b_{1}\left(R_{1}\right), t\right)=b_{2}\left(R_{1}\right), \quad k_{2} b_{2 r}\left(R_{1}\right)-k_{1} b_{1 r}\left(R_{1}\right)=æ b_{1}\left(R_{1}\right) v_{1}\left(R_{1}\right),
\end{gathered}
$$

where $\mu_{j}=\rho_{j} \nu_{j}$ is the dynamic viscosity coefficient. Moreover, functions $u_{1}(r), v_{1}(r), a_{1}(r)$ and $b_{1}(r)$ are bounded at $r=0$.

Let us note that the problem is non-linear and inverse problem because along with $v_{j}(r)$, $a_{j}(r), b_{j}(r)$, constants $f_{j}$ (pressure gradients along the layers) are also unknown. Excluding functions $u_{j}(r)$ from the second equations (11) and taking into account adhesion conditions on the wall, we obtain the adjoint problem for functions $v_{j}(r), a_{j}(r)$. For known $u_{j}(r), a_{j}(r)$ the problem for functions $b_{j}(r)$ is separated. Functions $d_{j}(r)$ are found upon integrating (13).

We introduce dimensionless functions and parameters

$$
\begin{gathered}
V_{j}(\xi)=\frac{R_{1}^{2}}{\mathrm{M} \chi_{1}} v_{j}(r), \quad A_{j}(\xi)=\frac{a_{j}(r)}{\alpha}, \quad F_{j}=\frac{R_{1}^{4}}{\mathrm{M} \chi_{1}^{2}} f_{j}, \quad \mathrm{M}=\frac{\Re \alpha R_{1}^{3}}{\mu_{2} \chi_{1}}, \\
\xi=\frac{r}{R_{1}}, \quad \operatorname{Pr}_{j}=\frac{\nu_{j}}{\chi_{j}}, \quad \chi=\frac{\chi_{1}}{\chi_{2}}, \quad \mu=\frac{\mu_{1}}{\mu_{2}}, \quad k=\frac{k_{1}}{k_{2}}, \quad \gamma=\frac{R_{2}}{R_{1}},
\end{gathered}
$$

where parameter $\mathrm{M}$ is the Marangoni number. Then the nonlinear conjugate inverse boundary 
value problem takes the form in dimensionless variables

$$
\begin{gathered}
\operatorname{Pr}_{1}\left(V_{1 \xi \xi}+\frac{1}{r} V_{1 \xi}\right)+\frac{\mathrm{M}}{\xi} V_{1 \xi} \int_{0}^{\xi} x V_{1}(x) d x-\mathrm{M} V_{1}^{2}+F_{1}=0 \\
A_{1 \xi \xi}+\frac{1}{\xi} A_{1 \xi}+\frac{\mathrm{M}}{\xi} A_{1 \xi} \int_{0}^{\xi} x V_{1}(x) d x-2 V_{1} A_{1}=0, \quad 0<\xi \leqslant 1 \\
\frac{\operatorname{Pr}_{2}}{\chi}\left(V_{2 \xi \xi}+\frac{1}{\xi} V_{2 \xi}\right)-\frac{\mathrm{M}}{\xi} V_{2 \xi} \int_{\xi}^{R_{2}} x V_{2}(x) d x-\mathrm{M} V_{2}^{2}+F_{2}=0, \\
\frac{1}{\chi}\left(A_{2 \xi \xi}+\frac{1}{\xi} A_{2 \xi}\right)-\frac{\mathrm{M}}{\xi} A_{2 \xi} \int_{\xi}^{R_{2}} x V_{2}(x) d x-2 V_{2} A_{2}=0, \quad 1 \leqslant \xi \leqslant \gamma, \quad A_{2}(\gamma)=1, \\
V_{2}(\gamma)=0, \quad \int_{1}^{\gamma} x V_{2}(x) d x=0, \\
\int_{0}^{1} x V_{1}(x) d x=0, \quad\left|A_{1}(0)\right|<\infty \\
V_{2 \xi}(1)-\mu V_{1 \xi}(1)=-2 A_{1}(1), \\
V_{1}(1)=V_{2}(1), \quad\left|V_{1}(0)\right|<\infty \\
A_{2 \xi}(1)-k A_{1 \xi}(1)=\mathrm{E} A_{1}(1) V_{1}(1), \\
A_{1}(1)=A_{2}(1), \quad \mid A_{1},
\end{gathered}
$$

where parameters $\operatorname{Pr}_{j}$ are Prandtl numbers, $\mathrm{E}=\mathfrak{x}^{2} \alpha R_{1}^{2} / \mu_{2} k_{2}$ is the parameter that determines the influence of internal interfacial energy on the motion of fluids inside the layers. Integral conditions (23) allow us to find unknown constants (pressure gradients along the layers) $F_{j}$, $j=1,2$.

\section{Solution of model problems}

Let us consider the obtained nonlinear conjugate inverse boundary value problem (20)-(27). Equations (20), (21) contain the Marangoni number. Let us assume that $\mathrm{M} \ll 1$, that is, we consider the creeping thermocapillary motion. First we exclude all nonlinear terms in equations (20), (21). Then we leave unchanged the only nonlinear boundary condition (26) and assume that $\mathrm{E}=\mathrm{O}(1)$. After some transformations we get

$$
\begin{aligned}
& V_{1}(\xi)=-\frac{1}{4} \frac{F_{1}}{\operatorname{Pr}_{1}} \xi^{2}+C_{1} \ln (\xi)+C_{2}, \\
& A_{1}(\xi)=C_{3} \ln (\xi)+C_{4}, \quad 0 \leqslant \xi \leqslant 1 ; \\
& V_{2}(\xi)=-\frac{1}{4} \frac{\chi F_{2}}{\operatorname{Pr}_{2}} \xi^{2}+C_{5} \ln (\xi)+C_{6}, \\
& A_{2}(\xi)=C_{7} \ln (\xi)+C_{8}, \quad 1 \leqslant \xi \leqslant \gamma,
\end{aligned}
$$

where $C_{i}(i=1, \ldots, 8)$ are unknown constants. Substituting solutions (28), (29) into the system of boundary conditions (22)-(27), we obtain an algebraic system of equations for unknown $C_{i}$ and $F_{j}$. Thus, boundedness of functions $V_{1}(\xi)$ and $A_{1}(\xi)$ at $\xi=0((25),(27))$ implies that $C_{1}=C_{3}=0$. Functions $A_{j}(\xi)$ are equal on the interface $(27)$ then $C_{4}=C_{8}$. Taking into 
account conditions $(22)-(24)$ and condition of equality of functions $V_{j}(\xi)$ on the interface, we obtain from (25) that

$$
\begin{gathered}
C_{2}=-\frac{2(S \ln (\gamma)-1)}{4 \mu(S \ln (\gamma)-1)+2 L+1} C_{8}, \quad C_{5}=-\frac{2}{4 \mu(S \ln (\gamma)-1)+2 L+1} C_{8}, \\
C_{6}=\frac{2\left(L \gamma^{2}+\ln (\gamma)\right)}{4 \mu(S \ln (\gamma)-1)+2 L+1} C_{8}, \\
F_{1}=-\frac{16 \operatorname{Pr}_{1}(S \ln (\gamma)-1)}{4 \mu(S \ln (\gamma)-1)+2 L+1} C_{8}, \quad F_{2}=\frac{8 \operatorname{Pr}_{2} L}{\chi(4 \mu(S \ln (\gamma)-1)+2 L+1)} C_{8},
\end{gathered}
$$

where

$$
L=\frac{1+2 \ln (\gamma)-\gamma^{2}}{\left(\gamma^{2}-1\right)^{2}}, \quad S=\frac{\gamma^{2}+1}{\gamma^{2}-1},
$$

and constant $C_{8}$ is the root of the quadratic equation

$$
\frac{2 \mathrm{E} \ln (\gamma)(S \ln (\gamma)-1)}{4 \mu(S \ln (\gamma)-1)+2 L+1} C_{8}^{2}+C_{8}-1=0
$$

It is clear that when there is no effect of internal interfacial energy on the motion of fluids inside the layers $(\mathrm{E}=0)$, that is, the heat fluxes on the interface are equal to each other or $\gamma \rightarrow 1$ (radii of the outer and inner cylinders coincide) equation (32) has one root $C_{8}=1$. In other cases when $\gamma>1$ and $\mathrm{E} \neq 0(\alpha \neq 0)$ equation (32) has two real roots. In general, the analysis depends on the sign of the expression

$$
D=1+\frac{8 \mathrm{E} \ln (\gamma)(S \ln (\gamma)-1)}{4 \mu(S \ln (\gamma)-1)+2 L+1} .
$$

For $D>0$ equation (32) has two roots and for $D=0$ it has one root. When $D<0$ problem (20)-(27) has no solutions.

Taking into account $(28),(29)$, unknown dimensionless velocity functions have the form

$$
\begin{aligned}
& U_{1}(\xi)= \frac{1}{16} \frac{F_{1}}{\operatorname{Pr}_{1}} \xi^{3}-\frac{1}{2} C_{2} \xi \\
& U_{2}(\xi)=-\frac{1}{16} \frac{F_{2}}{\operatorname{Pr}_{2}} \xi^{3}+\frac{1}{4}\left((2 \ln (\xi)-1) C_{5}+2 C_{6}\right) \xi- \\
& \quad-\frac{1}{16}\left(-\frac{\chi F_{2}}{\operatorname{Pr}_{2}} \gamma^{2}+4(2 \ln (\gamma)-1) C_{5}+8 C_{6}\right) \gamma^{2} \xi^{-1},
\end{aligned}
$$

where constants $C_{2}, C_{5}, C_{6}, F_{j}$ are from (30), (31). Taking into account (28)-(32), the remaining required functions $b_{j}(r), p_{j}(r, z), d_{j}(r)$ are determined from equations (12), (13).

We study the effect of changes in the internal energy of the interface on the velocity profiles $U_{j}(\xi)$ and functions $V_{j}(\xi)$. We choose water $\mathrm{H}_{2} \mathrm{O}$ and liquid $\mathrm{CO}_{2}$ as working medium. Parameters of liquids are given in the order " $\mathrm{H}_{2} \mathrm{O}, \mathrm{CO}_{2} ": \rho=\left\{0.998 \cdot 10^{3}, 1.977\right\} \mathrm{kg} / \mathrm{m}^{3}, \nu=\{1.004$. $\left.10^{-6}, 7.386 \cdot 10^{-6}\right\} \mathrm{m}^{2} / \mathrm{s}, \chi=\left\{1.442 \cdot 10^{-7}, 9.828 \cdot 10^{-6}\right\} \mathrm{m}^{2} / \mathrm{s}, \mathrm{k}=\left\{0.5984,1.643 \cdot 10^{-2}\right\} \mathrm{Vt} /(\mathrm{m} \cdot \mathrm{K})$, $æ=1.989 \cdot 10^{-4} \mathrm{~N} /(\mathrm{m} \cdot \mathrm{K}), \sigma_{0}=72.86 \cdot 10^{-3} \mathrm{~N} / \mathrm{m}$. Velocity profiles $\bar{U}_{j}(\xi)$ and functions $\bar{V}_{j}(\xi)$ are shown in Fig. 2 for various values of parameter $\mathrm{E}=\{0.01,0.02,0.1,0.7\}$, where $\bar{U}_{j}(\xi)=$ $\mathrm{M}^{-1} U_{j}(\xi)$ and $\bar{V}_{j}(\xi)=\mathrm{M}^{-1} V_{j}(\xi), \mathrm{M}=\mathrm{E} / \mathrm{M}_{0}, \mathrm{M}_{0}=æ \chi_{1} /\left(k_{2} R_{1}\right)$. 


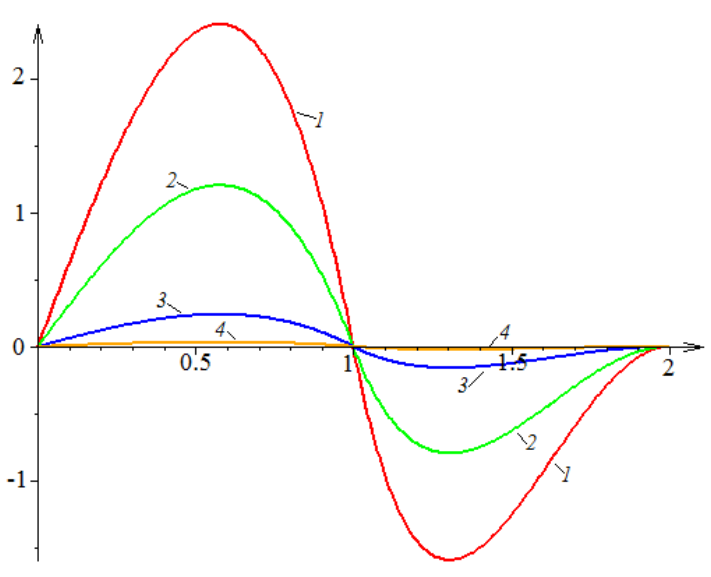

a) $\bar{U}_{j}(\xi)$

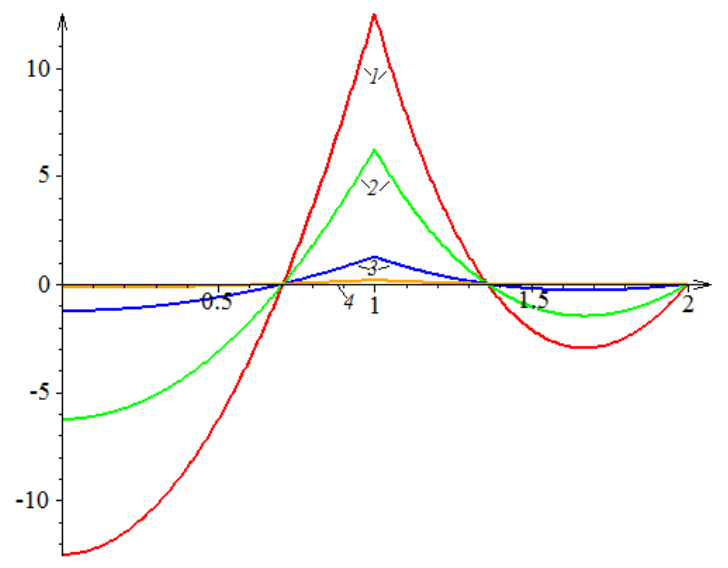

б) $\bar{V}_{j}(\xi)$

Fig. 2. The influence of parameter $\mathrm{E}$ on velocity profiles $\bar{U}_{j}(\xi)$ and functions $\bar{V}_{j}(\xi) .1-\mathrm{E}=0.01$, $2-\mathrm{E}=0.02,3-\mathrm{E}=0.1,4-\mathrm{E}=0.7$

Profiles of velocity $\bar{U}_{1}(\xi)$ and function $\bar{V}_{1}(\xi)$ are presented on the interval $0 \leqslant \xi \leqslant 1$. Profiles of velocity $\bar{U}_{2}(\xi)$ and function $\bar{V}_{2}(\xi)$ are presented on the interval $1 \leqslant \xi \leqslant \gamma$. It is also assumed that $R_{1}=10^{-10} \mathrm{~m}$, and the ratio of the outer radius and the inner radius of the cylinder remains constant $(\gamma=2)$. The Fig. 2 shows that with the increase of the internal energy parameter of the interphase boundary values of functions $\bar{U}_{j}(\xi)$ and $\bar{V}_{j}(\xi)$ decrease. In Fig. 3 profiles of velocity $\bar{U}_{j}(\xi)$ and functions $\bar{V}_{j}(\xi)$ are presented for various values of the internal radius of the cylinder $\left(R_{1}=\left\{10^{-10}, 2 \cdot 10^{10}, 5 \cdot 10^{10}, 10^{-9}\right\} \mathrm{m}\right)$ for $\gamma=2$ and $\mathrm{E}=0.7$. Here the Marangoni number is determined as $\mathrm{M}=æ \Theta R_{1} /\left(\mu_{2} \chi_{1}\right)$, where $\Theta=\alpha R_{1}^{2}=623.15 \mathrm{~K}$ that corresponds to the critical temperature for water. The Fig. 3 shows that functions $\bar{U}_{j}(\xi)$ and $\bar{V}_{j}(\xi)$ decrease with increasing $R_{1}$.

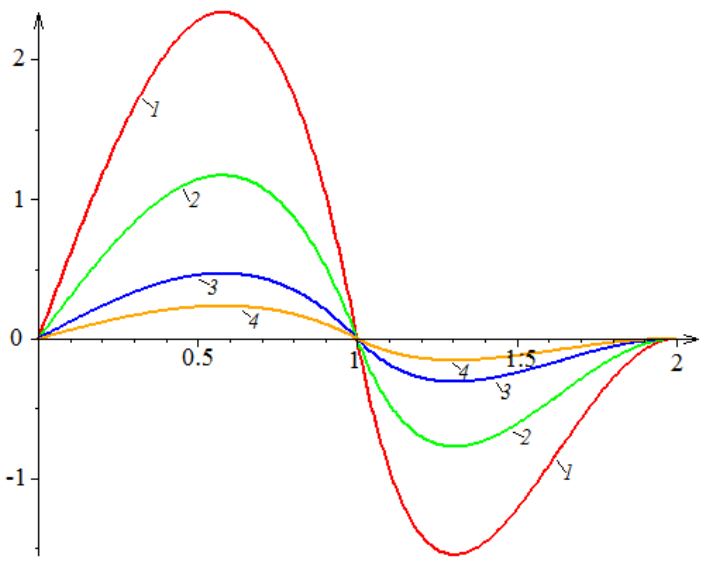

a) $\bar{U}_{j}(\xi)$

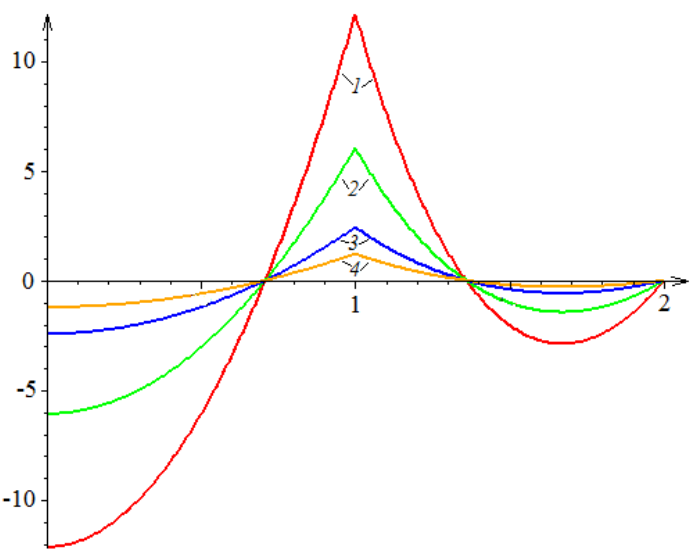

б) $\bar{V}_{j}(\xi)$

Fig. 3. The influence of parameter $R_{1}$ on profiles of velocity $\bar{U}_{j}(\xi)$ and functions $\bar{V}_{j}(\xi)$. 1 $R_{1}=10^{-10} \mathrm{~m}, 2-R_{1}=2 \cdot 10^{-10} \mathrm{~m}, 3-R_{1}=5 \cdot 10^{-10} \mathrm{~m}, 4-R_{1}=10^{-9} \mathrm{~m}$

This effect is due to the fact that the increase of the inner radius of the cylinder $R_{1}$ for fixed 
$\gamma=2$ the influence of the constant temperature defined on the outer surface of the cylinder weakens. Fig. 4 shows a relationship between profiles of velocity $\bar{U}_{j}(\xi)$, functions $\bar{V}_{j}(\xi)$ and $(\gamma)$ for $\mathrm{E}=0.7, \mathrm{M}=0.04, R_{1}=10^{-10} \mathrm{~m}$.

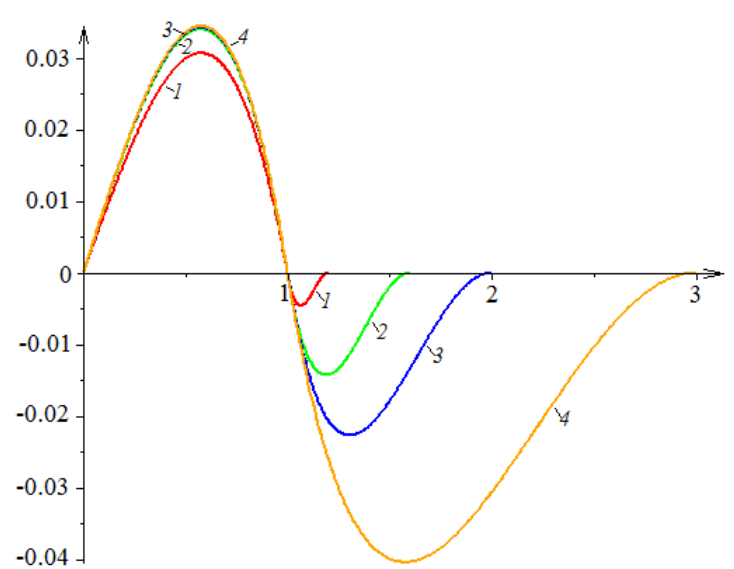

a) $\bar{U}_{j}(\xi)$

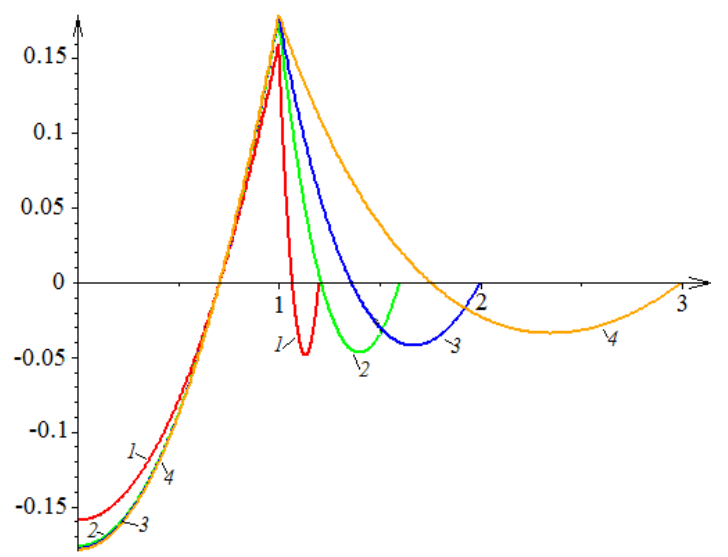

б) $\bar{V}_{j}(\xi)$

Fig. 4. The influence of parameter $\gamma$ on profiles of velocity $\bar{U}_{j}(\xi)$ and functions $\bar{V}_{j}(\xi)$. 1 $\gamma=1.2,2-\gamma=1.6,3-\gamma=2,4-\gamma=3$

It is clear that the increase of parameter $\gamma=\{1.2,1.6,2,3\}$ strongly affects profiles of velocity $\bar{U}_{2}(\xi)$ and function $\bar{V}_{2}(\xi)$. Values of these functions are significantly increased. The increase of values of functions $\bar{U}_{1}(\xi)$ and $\bar{V}_{1}(\xi)$ is not so significant. This can be explained by the fact that for a fixed value of $R_{1}$ the radius of the outer cylinder increases because $\gamma=R_{2} / R_{1}$.

Thus, the influence of the internal energy of the interface on the two-layer flow in the cylinder was studied. It was found that with the increase of parameter $\mathrm{E}$ values of functions $\bar{U}_{j}(\xi)$ and $\bar{V}_{j}(\xi)$ decrease.

This research was supported by the Russian Foundation for Basic Research (grant no. 17-0100229).

\section{References}

[1] A.Adamson, Physical chemistry of surfaces, Moscow, Mir, 1979.

[2] J.F.Harper, D.W.Moore, J.R.A.Pearson, The effect of the variation of surface tension with temperature on the motion of drops and bubbles, J. Fluid Mech., 27(1967), 361.

[3] V.K.Andreev, V.E.Zakhvatayev, E.A.Ryabitsky, Thermocapillary instability, Monograph, Novosibirsk, Nauka, 2000 (in Russian).

[4] F.E.Torres, E.Helborzheimer, Temperature gradients and drag effects produced by convection of interfacial internal energy around bubbles, Phys. Fluids A, 5(1993), no. 3, 537-549.

[5] V.K.Andreev, On the Friedrichs inequality for compound domains, J. Sib. Fed. Univ. Mat. and Fis., 2(2009), no. 2, 146-157 (in Russian). 
[6] V.K.Andreev, Yu.A.Gaponenko, Mathematical modeling of convective currents, Krasnoyarsk, KrasGU, 2006 (in Russian).

[7] V.K.Andreev, V.V.Puhnachev, Invariant solutions of the equations of thermocapillary motion, Numerical methods of continuum mechanics. Novosibirsk, 14(1983), no. 5, 3-23 (in Russian).

[8] G.Bateman, A.Erdein, Higher transcendental functions. Bessel functions, parabolic cylinder functions, orthogonal polynomials, Moscow, Nauka, 1974 (in Russian).

[9] V.S.Vladimirov, Equations of mathematical physics, Moscow, Nauka, 1976 (in Russian).

[10] S.G.Mikhlin, Linear Partial Differential Equations, Moscow, High school, 1977 (in Russian).

[11] L.V.Ovsyannikov, Group analysis of differential equations, Moscow, Nauka, 1978 (in Russian).

[12] A.P.Prudnikov, Yu.A.Bychkov, O.I.Marichev, Integrals and series. Special functions, Moscow, Nauka, 1983 (in Russian).

\section{Влияние изменений внутренней энергии поверхности раздела на двухслойное течение в цилиндре}

Евгений П. Магденко

Институт вычислительного моделирования СО РАН

Академгородок, 50/44, Красноярск, 660036 Институт математики и фундаментальной информатики Сибирский федеральный университет Свободный, 79, Красноярск, 660041

Россия

В данной работе исследуется точное решение для уравнений модели ползущего течения с полем скоростей типа Хименца, описывающее термокапиллярную конвекцию в слоях. Оно интерпретируется как движение вязких теплопроводных жидкостей в иилиндре с твердыми стенками и общей подвижной недеформируемой поверхностью раздела. При этом массовые силы отсутствуют. С математической точки зрения возникающая начально-краевая задача является обратной и нелинейной, так как учитывается полное энергетического условие на границе раздела. Установлено, что может существовать два таких решения.

Ключевые слова: нелинейная обратная задача, число Марангони, энергетическое условие, ползущее термокапиллярное движение, решение Химениа. 